\title{
Adaptação transcultural da versão original da Escala de Avaliação Sociofamiliar em Idosos para o contexto brasileiro
}

\author{
Cross-cultural adaptation of the original version of the Older Adults Socio-familial Evaluation \\ Scale to the Brazilian context
}

\author{
Luciana Shirley Pereira Zanela' ID \\ Jesús Vicente García Gonzalez² ${ }^{\text {ID }}$ \\ Albert Schiaveto de Souza ${ }^{3}$ ID \\ Samuel Leite Oliveira ${ }^{4}$ (D) \\ Alexandra Maria Almeida Carvalho' ${ }^{\mathbb{D}}$
}

\section{Resumo}

Objetivo: Traduzir e adaptar transculturalmente a escala espanhola de Avaliação Sociofamiliar em Idosos (Escala de Gijón) para o contexto brasileiro. Métodos: Procedimento metodológico de adequação transcultural, com tradução (espanhol-português), retrotradução (portuguêsespanhol), avaliação de equivalências semântica, idiomática, experimental e conceitual, e pré-teste da versão brasileira em uma amostra de 30 idosos. Para análise de concordância foram medidos proporcionalidade e índice Kappa Cohen-Fleiss ( $\kappa)$. Em adição, confirmouse a consistência interna pelo alfa de Cronbach. Resultados: A condução das traduções (T1 e T2), e retrotraduções (RT1 e RT2), e avaliações da versão síntese (T12) por comitê de especialistas e juiz neutro garantiram em T12 a essência avaliativa da versão original da escala. A avaliação dos 34 componentes de T12 mostrou adequação semântica (100\%) e idiomática (94\%), e adequação superior a 70\% para equivalências experimental e conceitual. Houve concordância quase-perfeita entre os especialistas do comitê: semântica $(\kappa=0,95)$, idiomática $(\kappa=0,97)$, experimental $(\kappa=0,98)$ e conceitual $(\kappa=0,99)$. O pré-teste de T12 resultou confiabilidade substancial para o instrumento, com alpha de Cronbach de 0,77. Conclusão: Este estudo garante a adaptação transcultural da escala de Avaliação Sociofamiliar em Idosos para o contexto da pessoa idosa que vive no Brasil. A avaliação das equivalências resultou em concordância quase-perfeita entre os especialistas. O públicoalvo não reportou dificuldades para compreender as assertivas da escala. Demonstrou-se

Palavras-chave: Estudos de Validação. Risco. Saúde do Idoso. Vulnerabilidade Social.

\footnotetext{
Universidade Federal de Mato Grosso do Sul, Faculdade de Medicina, Programa de Pós-graduação em Saúde e Desenvolvimento na Região Centro-Oeste. Campo Grande, MS, Brasil.

2 Servicio de Transformación y Gestión del Conocimiento; Consejería de Salud de Asturias. Oviedo, Asturias, España.

3 Universidade Federal de Mato Grosso do Sul, Instituto de Biociências, Pós-graduação em Saúde e Desenvolvimento na Região Centro-Oeste. Campo Grande, MS, Brasil.

4 Universidade Federal de Mato Grosso do Sul, Instituto de Física. Campo Grande, MS, Brasil.
}

Os autores declaram não haver conflito na concepção deste trabalho.

Financiamento: Coordenação de Aperfeiçoamento de Pessoal de Nível Superior - Brasil (CAPES), Código de Financiamento 001, sob processo n. 88882.458315/2019-01. 
um instrumento confiável, considerando o alfa de Cronbach obtido. Após a validação da escala, estudo em andamento, será disponibilizado um instrumento confiável para o rastreio da situação sociofamiliar da pessoa idosa no contexto brasileiro.

\section{Abstract}

Objective: To cross-culturally translate and adapt the Spanish Older Adults Socio-familial Evaluation Scale (Gijón Scale) to the Brazilian context. Methods: A methodological procedure of cross-cultural adaptation with translation (Spanish-Portuguese), back-translation (Portuguese-Spanish), evaluation of semantic, idiomatic, experimental, and conceptual equivalences, and pretest of the Brazilian version in a sample of 30 older adults. For the concordance analysis, proportionality and the Kappa Cohen-Fleiss index ( $\kappa)$ were measured. In addition, internal consistency was confirmed by Cronbach's alpha. Results: Translations (T1 and T2), back-translations (RT1 and RT2), and evaluations of the synthesis version (T12) were carried out by a Committee of Experts, and a neutral judge ensured the evaluative essence of the original version of the scale in T12. The evaluation of the 34 components of T12 showed semantic (100\%) and idiomatic (94\%) adequacy, and adequacy greater than $70 \%$ for experimental and conceptual equivalences. There was almost perfect concordance among the experts of the Committee: semantics $(\kappa=0.95)$, idiomatic $(\kappa=0.97)$, experimental $(\kappa=0.98)$, and conceptual $(\kappa=0.99)$. The T12 pretest resulted in substantial reliability of the instrument with a Cronbach's alpha of 0.77 . Conclusion: The present study ensures the cross-cultural adaptation of the Socio-familial Evaluation Scale to the context of the older person living in Brazil. The equivalence evaluation resulted in almost perfect concordance among experts. The target audience did not report difficulties in understanding the assertions of the scale. The instrument proved to be reliable considering the Cronbach's alpha obtained. After validating the scale, an ongoing study, a reliable instrument will be made available for tracking the socio-familial situation of older adults in the Brazilian context.
Keywords: Validation Study. Risk. Health of the Elderly. Social Vulnerability.

\section{INTRODUÇÃO}

Recentes entendimentos apontam para a necessidade de ações interdisciplinares pautadas na interação entre os saberes específicos contidos nas diferentes ciências, no sentido de otimizar a atenção à pessoa idosa ${ }^{1-3}$. As características como o ambiente familiar ${ }^{4}$, o gênero e a etnia devem ser consideradas na avaliação do processo de saúdedoença, pois o desiquilíbrio entre esses fatores pode resultar em iniquidades ou desigualdades na saúde experienciadas pelo indivíduo no curso da vida ${ }^{5}$. A inclusão dos fatores psicossociais nas avaliações em saúde favorecem intervenções mais abrangentes ${ }^{6,7}$, planejamento de ações que podem ser de cunho preventivo, reabilitador e terapêutico ${ }^{8}$, impactando nas condições da saúde da população e no aproveitamento dos investimentos feitos pelo sistema de saúde?.
Contudo, a associação de outras dimensões voltadas à globalidade da atenção ainda divide os interesses da comunidade acadêmica ${ }^{9-11}$. A temática relacionada aos aspectos cognitivos e funcionais se destaca entre as investigações, em especial estudos que visam garantir a adequação de instrumentos para rastrear o perfil de idosos que vivem no Brasil ${ }^{12}$. Esse direcionamento aponta a hierarquização da atenção, com enfoque na avaliação funcional cognitiva e física, sendo um marcador de partida do modelo de saúde brasileiro ${ }^{13}$. O atendimento integrado, capaz de abranger a tríade formada pelos aspectos individual, social e programático da vida dessa população ${ }^{14-16}$, incide sobre possíveis quadros de vulnerabilidade social decorrentes do processo de envelhecimento ${ }^{17,18}$.

As condições e qualidade de vida de pessoas idosas relacionam-se ao seu contexto social e incidem sobre sua estrutura social e econômica ${ }^{4,19}$. Portanto, a 
multidimensionalidade na atenção, desde a avaliação inicial até o acompanhamento do indivíduo, estimula o seu protagonismo e de sua rede de relações e suporte social ${ }^{20}$. A avaliação destas dimensões específicas pode ser realizada com o suporte de diferentes instrumentos que atenderam os critérios de desenvolvimento e validação para uso no Brasil ${ }^{12,21,22}$. Porém, a avaliação dos aspectos sociais na área do envelhecimento humano ainda dispõe de abordagem reducionista $^{12,20}$, com a gestão de ações fragmentadas e desconectadas entre os prestadores de cuidados. Ademais, ressalta-se que os indicadores oriundos das avaliações dos aspectos sociais possibilitam a estruturação de políticas públicas e ações mais eficientes e abrangentes na atenção às demandas da população idosa?.

Estabelecer relação entre os fatores ambientais e socioeconômicos garante a ampliação das intervenções sobre o nível de coesão social e familiar assim como outros fatores individuais que podem estar associados à morbidade e à mortalidade dessa população ${ }^{7,20}$. Entretanto, para essa medida, é necessário o uso de um instrumento abrangendo diferentes dimensões sociais ${ }^{14,15}$.

Na avaliação do contexto social da vida do idoso, essas dimensões relacionam-se aos seguintes aspectos: ajuste ambiental, a adaptação pessoal, o bem-estar subjetivo e suas interações sociais ${ }^{15}$. A mensuração dessas dimensões é possível a partir da Escala de Avaliação Sociofamiliar em Idosos (EASFI), também conhecida como Escala de Gijón ${ }^{15}$. Em sua organização interna, avalia a situação sociofamiliar do/a entrevistado/a em caráter individual, apontando a situação social em três cortes distintos ${ }^{14-16}$.

Originalmente, construída e validada na Espanha, utilizada como uma ferramenta gerontológica, multidimensional para fins de rastreio social com confiabilidade substancial e boa fidedignidade ${ }^{15}$. Em Portugal, a escala foi submetida ao estudo de validação, compondo protocolos de rastreio gerontológico ${ }^{14}$. No Peru, foi adequada, sem o critério de validação, e integra o protocolo de "Valoración Clínica del Adulto Mayor"16. Em outros países de língua espanhola, a escala também é utilizada em investigações voltadas ao idoso, sem ter passado pelo processo de validação ${ }^{23-25}$.
Mesmo sem ter sido submetida ao rigor metodológico para fins de adaptação e validação de instrumentos avaliativos, a escala é utilizada no Brasil para avaliações em serviços na modalidade de Cuidados Continuados Integrados $(\mathrm{CCI})^{26}$. $\mathrm{O}$ uso da escala por assistentes sociais da equipe multidisciplinar do CCI, possibilitou a detecção de lacunas culturais e de linguagem, que motivaram o interesse pelo desenvolvimento desta pesquisa que consiste na adaptação transcultural (ATC) e posterior validação.

Assim, urge a ampliação do rol de instrumentos avaliativos para o contexto de idosos vivendo no Brasi ${ }^{12,21,22}$ Para essa finalidade, este trabalho tem como objetivo realizar a tradução e adaptação transcultural da Escala de Avaliação Sociofamiliar em Idosos para o contexto do idoso que reside no Brasil. Cabe destacar que a tradução e a ATC, representam as etapas iniciais de validação desta escala conferindo atributos e dimensões adequadas às variáveis do instrumento ${ }^{27}$. Com a adequação e validação da EASFI para o contexto brasileiro será possível levantar novos indicadores em saúde e estabelecer relação entre a condição de vida com o processo de saúde e doença de idosos ${ }^{14,15}$.

\section{MÉTODO}

A Escala de Avaliação Sociofamiliar em Idosos (EASFI) avalia a situação sociofamiliar do idoso em uma perspectiva multidimensional. Os estudos de validação conduzidos na Espanha e em Portugal relatam que a escala pode ser autoaplicável ou conduzida, preferencialmente, por profissional assistente social ${ }^{14,15}$.

Dentre suas características internas, a escala é organizada em cinco domínios que medem diferentes aspectos da vida do idoso: I- Situação familiar; II- Situação econômica; III- Situação da moradia; IV- Relações sociais; V- Rede de suporte social $^{14,15}$. Cada domínio é composto por cinco assertivas com valores de um a cinco. $\mathrm{O}$ entrevistador seleciona apenas uma assertiva por domínio. A soma dos valores atribuídos a cada assertiva assinalada resulta na pontuação global, organizada em três intervalos distintos: situação social adequada (5 a 9 
pontos), existência de risco social (10 a 14 pontos) ou existência de problema social (acima de 15 pontos) ${ }^{15}$. Este estudo apresenta a adaptação transcultural (ATC) da EASFI para o contexto da pessoa idosa vivendo no Brasil.

Estudos metodológicos destinam-se a obter, organizar e analisar dados e possibilitam a elaboração, validação ou avaliação de instrumentos e técnicas de pesquisa ${ }^{27-29}$. A ATC compreende essa modalidade de investigação, ao passo que mensura a precisão dos resultados e reduz os riscos de distorções de informações e interpretações durante o manuseio da escala ${ }^{27}$.

Para utilização da versão original, obteve-se autorização prévia do autor principal do estudo de validação. Além disso, o mesmo aprovou a versão adaptada transculturalmente ${ }^{27}$.

Conforme elencado na Figura 1, executou-se etapas distintas para a adaptação adequada da escala, considerando países com diferenças culturais e/ou de idioma ${ }^{27}$.

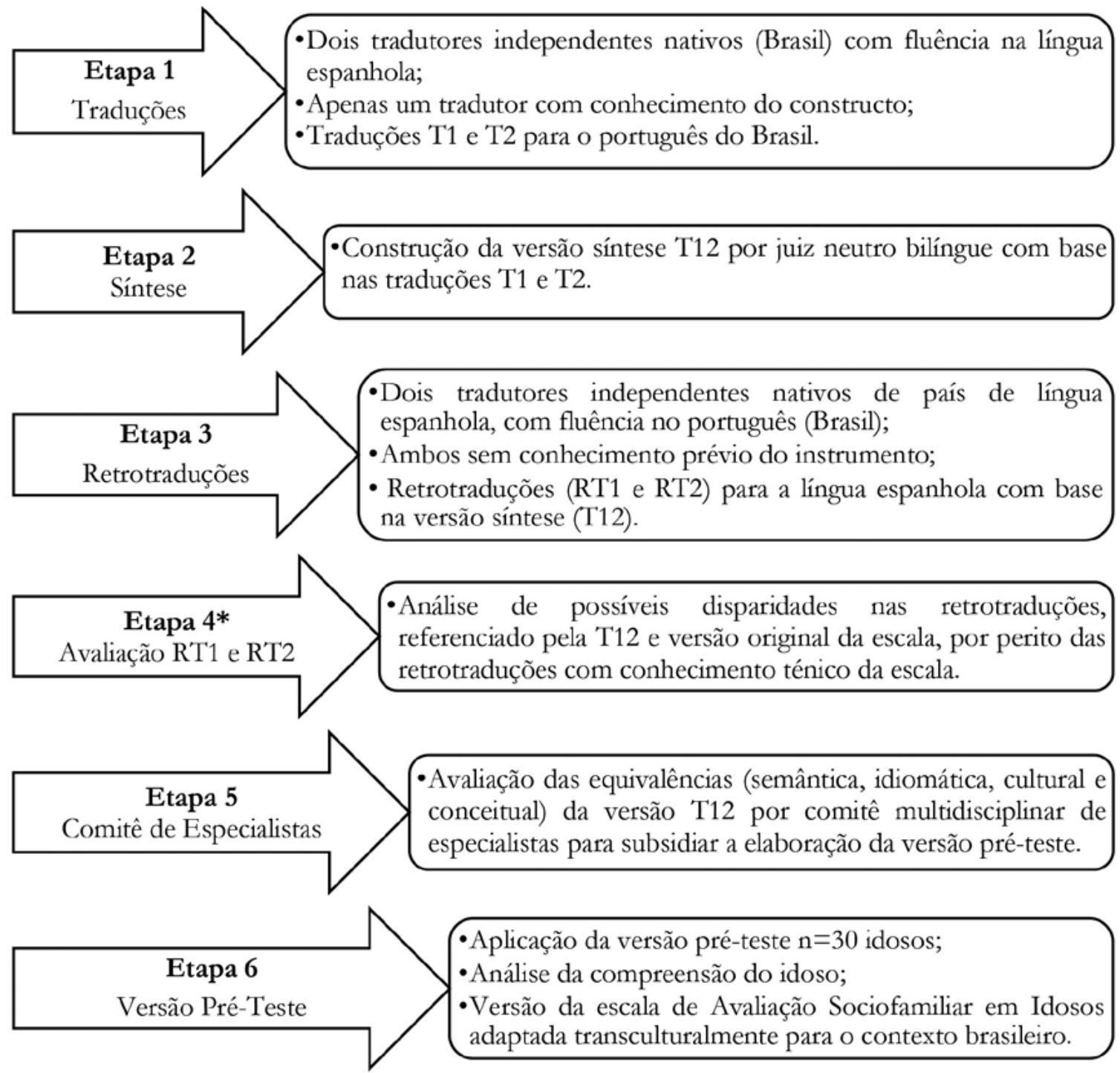

Figura 1. Etapas para Adaptação Transcultural da Escala de Avaliação Sociofamiliar em Idosos. 
Em conformidade com o método ${ }^{27}$ adotado neste estudo, as cinco etapas para a ATC foram cumpridas e uma etapa foi acrescentada. Em cada uma das etapas, os 34 componentes da escala foram submetidos a ATC: título da escala (1 item); domínios (5 itens); assertivas (25 itens); pontuação (3 itens).

A análise dos dados foi realizada com suporte do SPSS versão 23. Para a verificação da homogeneidade e das dimensões dos itens da escala na versão préteste, adotou-se o coeficiente alfa de Cronbach ${ }^{29,30}$.

\section{Etapa 1 - Tradução}

Participaram da primeira etapa dois tradutores, brasileiros com proficiência na língua espanhola. As traduções ocorreram de forma independente, resultando nas versões T1 e T2. Cada componente da escala foi traduzido e os comentários dos tradutores foram transcritos.

O intérprete responsável por T1 foi informado previamente sobre a pesquisa e sobre o objeto do estudo. O intérprete da versão T2 conduziu a tradução sem conhecimento da escala, mantendo o foco na linguagem, prevenindo efeitos ambíguos ao instrumento original, talvez não identificados pelo primeiro tradutor ${ }^{27}$.

\section{Etapa 2 - Síntese}

Na segunda etapa foi elaborada a versão síntese, denominada T12. Um juiz neutro, com proficiência na língua espanhola, observou o nível de concordância de acordo com o seguinte critério:

- Concordância total: traduções idênticas;

- Concordância parcial: alterações de até duas palavras na frase traduzida;

- Diferença entre as traduções: situações de ambiguidade, conflitos conceituais e/ou culturais entre as traduções.

O resultado dessa observação possibilitou que o juiz neutro selecionasse a tradução de maior consistência idiomática e cultural ou propusesse alterações na adequação do instrumento para o contexto do idoso vivendo no Brasil. Para finalização da versão T12, uma das seguintes ações foram praticadas pelo juiz neutro: sugestão de nova tradução; escolha por T1; escolha por T2; mantida T1 e T2 (quando idênticas); fundir T1 e T2.

\section{Etapa 3 - Retrotradução}

$\mathrm{Na}$ terceira etapa, T12 foi submetida à retrotradução. Participaram dois intérpretes, bilíngues, nativos de país com língua espanhola, residentes no Brasil a mais de 10 anos. As retrotraduções ocorreram de forma cega e independente, sem conhecimento da escala e de sua organização interna. Assim, obtevese duas versões (RT1 e RT2) retrotraduzidas para a língua espanhola ${ }^{27}$.

\section{Etapa 4 - Avaliação das retrotraduções}

A quarta etapa foi incluída, embora não prevista na metodologia original, visando garantir a equivalência semântica em T12, constatando sua validade, inconsistências e erros conceituais. Um perito, com conhecimento substancial da escala em sua versão original avaliou RT1 e RT2.

Essa avaliação determinou a adequação ou inadequação das retrotraduções tendo como referência T12 e a escala em sua versão original. Quando constatadas disparidades conceituais e/ou gramaticais em alguma retrotradução, o avaliador selecionou a versão mais adequada considerando o objeto principal do constructo.

\section{Etapa 5 - Comitê de Especialistas}

A etapa 5 foi conduzida pelo Comitê de Especialistas, composto por oito profissionais de diferentes áreas: metodologista, linguista, psicólogo, assistente social, médico e enfermeira. Conduziram as avaliações amparados pelo "Compilado Avaliativo", documento com as versões produzidas nas etapas anteriores e uma revisão teórica relacionada a cada componente da escala. Esta avaliação originou a versão submetida ao pré-teste. 
Os especialistas avaliaram a versão T12 em busca das equivalências semântica, idiomática, cultural e conceitual dos 34 componentes da escala, atribuindo as notas: (1) - extrema adequação sem necessidade de qualquer alteração, (0) - adequação com necessidade de alteração e (-1) - inadequação. É esperada proporção igual ou superior a $80 \%{ }^{31}$, para concordância adequada entre os especialistas. Para a avaliação da concordância de T12 foi adotado o coeficiente Kappa de Cohen-Fleiss $(\kappa)^{29}$, considerando os níveis: inexistente $(<0)$, leve $(0-0,2)$, moderada $(0,21-0,4)$, forte $(0,41-0,60)$, muito forte $(0,61-0,80)$, quase perfeita $(0,81-0,99)$ e perfeita $(1)^{32}$.

\section{Etapa 6 - Pré-teste}

O objetivo do pré-teste foi verificar a compreensão do instrumento por parte dos respondentes, bem como possíveis dificuldades com a aplicação da escala pelo entrevistador.

Nesta etapa foram utilizados três instrumentos: a versão pré-teste (T12), um formulário para análise da compreensão da escala pela pessoa entrevistada, e um questionário para coleta de dados sociodemográficos.

O pré-teste foi aplicado a um grupo de $30 \mathrm{idosos}^{27}$, compondo uma amostra do tipo não probabilística e por julgamento.

Foram incluídos idosos de 60 anos ou mais, de ambos os sexos, que estavam em atendimento no setor de ambulatório ou na unidade de internação do Hospital São Julião de Campo Grande (MS) no período da coleta de dados (outubro-novembro de 2017).
Esta pesquisa atende as normas de pesquisa com seres humanos descritas nas Resoluções $n^{\circ}$ 466/2012 e no 510/2016. O estudo foi avaliado e aprovado pelo Comitê de Ética de Pesquisa com Seres Humanos da Universidade Federal de Mato Grosso do Sul (UFMS), inscrito sob parecer n. 58735616.8.0000.0021. A utilização da Escala de Avaliação Sociofamiliar em Idosos (versão espanhola) foi autorizada pelo primeiro autor do estudo de validação via correio eletrônico.

A inclusão do idoso no rol de entrevistados ocorreu após leitura e assinatura do Termo de Consentimento Livre e Esclarecido (TCLE). As entrevistas foram conduzidas de modo a garantir o sigilo de suas respostas e a não interferência no atendimento.

\section{RESULTADOS}

Traduções e Versão Síntese

Inicialmente, a Escala de Avaliação Sociofamiliar em Idosos (versão espanhola) foi traduzida para o português do Brasil por dois tradutores independentes, e resultou nas duas versões (T1 e T2). A comparação entre os 34 componentes traduzidos, demonstrou $53 \%$ de concordância total entre os tradutores, $29,4 \%$ de concordância parcial e 17,6\% de diferença entre as traduções.

Posteriormente, o juiz neutro avaliou T1 e T2, alterando ou apontando a tradução mais apropriada para cada componente, resultando na síntese (T12). A organização desta versão foi norteada pelos aspectos de linguagem, contexto avaliativo da escala e realidade do idoso brasileiro. O Quadro 1 relata as ações do juiz neutro em relação a cada componente traduzido, primando por uma versão de melhor equivalência ao português falado no Brasil. 
Quadro 1. Descritivo das ações do juiz neutro na avaliação das traduções para elaboração da versão T12 (n=34). Campo Grande, MS, 2017.

\begin{tabular}{|c|c|c|c|c|c|}
\hline Descrição & $=$ & $\lambda^{\prime}$ & $8 ;$ & $\mathrm{T} 1$ & $\mathrm{~T} 2$ \\
\hline Nome da Escala & 1 item & - & - & - & - \\
\hline $5-9$ pontos & 1 item & - & - & - & - \\
\hline $10-14$ pontos & 1 item & - & - & - & - \\
\hline Acima de 15 pontos & 1 item & - & - & - & - \\
\hline Título dos domínios & 5 itens & - & - & - & - \\
\hline Assertivas Domínio A & - & A.2; A.3 & A.1; A.5 & - & A. 4 \\
\hline Assertivas Domínio B & B. 4 & B.3 & B.2; B. 5 & B.1 & - \\
\hline Assertivas Domínio C & - & - & C.1; C.2; C.3; C.4 & C. 5 & - \\
\hline Assertivas Domínio D & D.1; D.2; D.3;D.4 & - & - & - & D. 5 \\
\hline Assertivas Domínio E & - & - & D.1; D.2; D.3; D.5 & E.4 & - \\
\hline Total & 14 itens & 3 itens & 12 itens & 3 itens & 2 itens \\
\hline
\end{tabular}

(=) Traduções idênticas; (حઢ) Combinação entre as duas traduções; (C) Sugestão do juiz neutro alterando as traduções; (T1) Escolha pela tradução T1; (T2) Escolha pela tradução T2.

Constatou-se a manutenção de 14 itens (41\%) por serem componentes idênticos. As traduções divergentes foram tratadas de duas formas pelo juiz neutro. A combinação de T1 e T2 ocorreu para 3 $(8,9 \%)$ componentes. A escolha de T1 para outros $3(8,9 \%)$ itens e de T2, para 2 itens (5,9\%). Nestes casos, a versão escolhida representou adequadamente os aspectos de linguagem pautados no contexto avaliativo da escala. O juiz neutro sugeriu ajustes em $12(35,3 \%)$ componentes traduzidos.

\section{Retrotraduções}

As retrotraduções possibilitaram a comparação entre o instrumental original e a versão T12. Frente aos 34 componentes retrotraduzidos em RT1 e RT2, verificou-se $58,8 \%$ de concordância total entre os intérpretes, $32,4 \%$ de concordância parcial e somente $3(8,8 \%)$ componentes apresentaram diferença nas retrotraduções. Portanto, em comparação ao conteúdo da versão original da escala, constata-se que T12 está em conformidade com a essência avaliativa proposta.

\section{Avaliação das Retrotraduções}

O perito avaliou RT1 e RT2 tendo por referência a versão T12 e a escala original na versão espanhola. O Quadro 2 apresenta a avaliação das retrotraduções pelo perito. Quando constatadas disparidades conceituais e/ou gramaticais em algum componente das retrotraduções ou síntese, a versão mais adequada foi selecionada considerando o objetivo principal do constructo. No caso das retrotraduções idênticas, o perito avaliou que $70,6 \%$ delas atendiam aos objetivos do constructo. No caso das retrotraduções conflitantes, RT2 foi escolhida na maioria das vezes $(17,6 \%)$ em detrimento da RT1 $(8,8 \%)$.

Vale destacar que o perito indicou a assertiva A.2 (Domínio A) da versão T12, como versão de maior equivalência, quando comparada à RT1, RT2 e a versão original.

Concluída essa etapa, retomou-se a discussão com o juiz neutro para conclusão de T12 e finalização do Compilado Avaliativo para encaminhamento ao Comitê de Especialistas. 
Quadro 2. Avaliação das retrotraduções em relação a versão T12 e a versão original da Escala de Avaliação Sociofamiliar em Idosos (n=34). Campo Grande, MS, 2017.

\begin{tabular}{|c|c|c|c|}
\hline Descrição & RT1 & RT2 & Avaliação \\
\hline Nome da escala & A & A & RT1 e RT2 \\
\hline Intervalo de pontuação: 5 - 9 pontos & A & A & RT1 e RT2 \\
\hline Intervalo de pontuação: 10 - 14 pontos & A & A & RT1 e RT2 \\
\hline Intervalo de pontuação: Acima de 15 pontos & A & A & RT1 e RT2 \\
\hline Domínio A & A & A & RT1 e RT2 \\
\hline Assertiva A.1 & A & A & RT2 \\
\hline Assertiva A.2 & A & A & T12 \\
\hline Assertiva A.3 & $\mathrm{I}$ & A & RT2 \\
\hline Assertiva A.4 & I & A & RT2 \\
\hline Assertiva A.5 & A & A & RT2 \\
\hline Domínio B & A & A & RT1 e RT2 \\
\hline Assertiva B.1 & A & A & RT1 e RT2 \\
\hline Assertiva B.2 & A & A & RT1 e RT2 \\
\hline Assertiva B.3 & A & A & RT1 e RT2 \\
\hline Assertiva B.4 & A & A & RT1 e RT2 \\
\hline Assertiva B.5 & A & A & RT1 e RT2 \\
\hline Domínio C & $\mathrm{A}$ & $\mathrm{A}$ & RT1 е RT2 \\
\hline Assertiva C.1 & A & A & RT1 \\
\hline Assertiva C.2 & A & A & RT1 \\
\hline Assertiva C.3 & A & A & RT1 e RT2 \\
\hline Assertiva C.4 & A & A & RT1 e RT2 \\
\hline Assertiva C.5 & A & A & RT1 e RT2 \\
\hline Domínio D & A & A & RT1 e RT2 \\
\hline Assertiva D.1 & A & A & RT1 e RT2 \\
\hline Assertiva D.2 & A & A & RT1 e RT2 \\
\hline Assertiva D.3 & A & A & RT1 e RT2 \\
\hline Assertiva D.4 & A & A & RT2 \\
\hline Assertiva D.5 & A & A & RT1 е RT2 \\
\hline Domínio E & A & A & RT1 e RT2 \\
\hline Assertiva E.1 & A & A & RT1 e RT2 \\
\hline Assertiva E.2 & A & A & RT1 \\
\hline Assertiva E.3 & A & A & RT1 e RT2 \\
\hline Assertiva E.4 & A & A & RT2 \\
\hline Assertiva E.5 & A & A & RT1 e RT2 \\
\hline
\end{tabular}

A=Adequado; I=Inadequado; T12=Síntese; RT1=Retrotradução 1; RT2=Retrotradução 2. 


\section{Comitê de Especialistas}

Como diretriz para analisar as versões T1, T2, T12, RT1 e RT2, o Comitê de Especialistas sugeriu que não se adotasse metáforas, linguagens coloquiais ou substantivos e verbos com sentido ambíguo, para otimizar a compreensão dos componentes da escala. Alterações ou substituições sugeridas pelo Comitê se deram pelo estudo de cada componente, com base no Compilado Avaliativo.

A proporção de concordância entre os especialistas na avaliação de adequação dos componentes da versão a ser submetida para pré-teste, foi superior a $80 \%$ tanto na equivalência semântica (100\%) quanto na idiomática (94\%). Por sua vez, as equivalências experimental e conceitual apresentaram proporção de concordância de $71 \%$ e $73 \%$, respectivamente. Por fim, em busca de garantir as equivalências experimental e conceitual, a versão síntese foi reavaliada pelos membros do Comitê para a consolidação da versão submetida ao pré-teste. Os índices kappa associados a essa versão indicam concordância quase-perfeita ${ }^{32}$ entre as avaliações semântica $(\kappa=0,95)$, idiomática $(\kappa=0,97)$, experimental $(\kappa=0,98)$ e conceitual $(\kappa=0,99)$.

\section{Pré-teste}

Durante a aplicação da versão T12 no pré-teste, observou-se objetividade e clareza das respostas dos entrevistados. O tempo médio de aplicação da escala foi de 7 minutos.

Para avaliação da compreensão dos idosos em relação ao instrumento, procedeu-se a leitura de cada assertiva da escala por parte do entrevistador. $\mathrm{Na}$ sequência, foi solicitado ao entrevistado interpretação do item e a confirmação ou negativa de compreensão clara do componente apresentado. A totalidade dos participantes relatou compreensão total, não reportando dificuldades para compreender as assertivas da escala.

Dentre os 30 idosos participantes do pré-teste, $60 \%$ (18) declararam tempo de escolarização inferior a 3 anos ou nunca ter frequentado a escola.

A avaliação sociofamiliar por meio do instrumento indicou que $66,7 \%$ dos idosos apresentavam situação social adequada ou aceitável.

O coeficiente de Cronbach total para os resultados da aplicação da versão pré-teste indica consistência interna substancial. Tendo como referência o intervalo de 0,61-0,80 para consistência interna substancial ${ }^{29,30}$, a exclusão de qualquer um dos domínios não impacta a interpretação da confiabilidade total da escala que apresentou o valor de alfa total de 0,77 (Tabela 1).

Após finalizada todas as etapas do processo de ATC, a Escala de Avaliação Sociofamiliar em Idosos adaptada para o contexto brasileiro foi analisada e aprovada unanimemente pelo Comitê de Especialistas e pelo autor principal da versão espanhola. Assim, não foi necessário proceder uma revisão e novo pré-teste.

A versão brasileira da EASFI (Quadro 3) subsidia a pesquisa em andamento para a validação e aferição de suas propriedades psicométricas.

Tabela 1. Resultados do desvio padrão, média e alfa de Cronbach em caso de exclusão de algum domínio avaliativo $(\mathrm{n}=30)$. Campo Grande, MS, 2017.

\begin{tabular}{llll}
\hline Domínios Avaliativos & Desvio Padrão & $\begin{array}{l}\text { Média de escala } \\
\text { se o item for excluído }\end{array}$ & $\begin{array}{l}\text { Alfa de Cronbach } \\
\text { se item excluído }\end{array}$ \\
\hline Domínio A $^{(1)}$ & 1,2 & 7,57 & 0,77 \\
Domínio B $^{(2)}$ & 0,8 & 6,40 & 0,82 \\
Domínio C $^{(3)}$ & 0,9 & 8,33 & 0,70 \\
Domínio D $^{(4)}$ & 1,1 & 8,00 & 0,66 \\
Domínio E $^{(5)}$ & 0,8 & 8,50 & 0,65 \\
\hline Total da escala & 1,21 & 9,7 & 0,77 \\
\hline
\end{tabular}

(1) Situação Familiar; ${ }^{(2)}$ Situação Econômica; ${ }^{(3)}$ Situação da Moradia; (4) Relações Sociais; ${ }^{(5)}$ Rede de Suporte Social. 
Quadro 3. Escala de Avaliação Sociofamiliar em Idosos (Versão brasileira) na versão pré-teste. Campo Grande (MS), 2018.

\begin{tabular}{|c|c|}
\hline \multirow{2}{*}{$\begin{array}{l}\text { Componente } \\
\text { Domínio A }\end{array}$} & Descritivo \\
\hline & Situação Familiar \\
\hline Assertiva A.1 & Mora com a família sem apresentar dependência física nem psíquica \\
\hline Assertiva A.2 & $\begin{array}{l}\text { Mora somente com o cônjuge de idade aproximada, sem apresentar dependência física nem } \\
\text { psíquica }\end{array}$ \\
\hline Assertiva A.3 & Mora com a família (e/ou cônjuge) e apresenta algum grau de dependência física ou psíquica \\
\hline Assertiva A.4 & Mora sozinho e tem filho(s) que reside(m) perto \\
\hline Assertiva A.5 & Mora sozinho e não tem filho(s) ou este(s) reside(m) longe \\
\hline Domínio B & Situação Econômica \\
\hline Assertiva B.1 & Renda individual acima de 2,4 vezes o salário-mínimo \\
\hline Assertiva B.2 & Renda individual a partir de 1,3 até 2,4 vezes o salário-mínimo \\
\hline Assertiva B.3 & Renda individual a partir de 1 até 1,3 vezes o salário-mínimo \\
\hline Assertiva B.4 & Renda individual proveniente de BPC/LOAS \\
\hline Assertiva B.5 & Sem renda ou renda individual inferior a 1 salário-mínimo \\
\hline Domínio C & Situação da Moradia \\
\hline Assertiva C.1 & $\begin{array}{l}\text { Moradia adequada às necessidades do idoso (saneamento básico, rede de abastecimento de } \\
\text { água, rede de abastecimento de energia elétrica e acessibilidade) }\end{array}$ \\
\hline Assertiva C. 2 & Moradia com barreiras arquitetônicas (escadas, degraus, portas estreitas etc.) \\
\hline Assertiva C.3 & $\begin{array}{l}\text { Moradia com higiene precária, umidade (mofo e fungos), ventilação e iluminação } \\
\text { inadequadas }\end{array}$ \\
\hline Assertiva C.4 & Moradia sem telefone e/ou sem elevador (em caso de sobrado ou apartamento) \\
\hline Assertiva C.5 & $\begin{array}{l}\text { Moradia inadequada (declarada em ruínas, barracos, cortiços etc.) e/ou ausência de } \\
\text { infraestrutura básica (rede de abastecimento de água, rede de abastecimento de energia } \\
\text { elétrica ou rede de esgoto/fossa séptica) ou em situação de rua }\end{array}$ \\
\hline Domínio D & Relações Sociais \\
\hline Assertiva D.1 & Relação social com rede de apoio (família, vizinhos, trabalho, amizades, comunidade etc.) \\
\hline Assertiva D.2 & Relação social somente com família e vizinhos \\
\hline Assertiva D.3 & Relação social somente com a família ou somente com os vizinhos \\
\hline Assertiva D.4 & Não sai da moradia, mas recebe visitas \\
\hline Assertiva D.5 & Não sai da moradia e não recebe visitas ou sem relação social com rede de apoio \\
\hline Domínio E & Rede de Suporte Social \\
\hline Assertiva E.1 & Recebe suporte social da família ou de vizinhos \\
\hline Assertiva E.2 & Recebe em domicílio atendimento de voluntariado, da rede pública e/ou da rede privada \\
\hline Assertiva E.3 & $\begin{array}{l}\text { Não tem rede de suporte social, mas tem condições de permanecer em casa e realizar o } \\
\text { autocuidado }\end{array}$ \\
\hline Assertiva E.4 & Necessita ingressar em ILPI por situação de vulnerabilidade social \\
\hline Assertiva E.5 & $\begin{array}{l}\text { Necessita de cuidados permanentes em ILPI para atividades básicas e instrumentais da vida } \\
\text { diária }\end{array}$ \\
\hline
\end{tabular}

\begin{tabular}{|c|c|}
\hline \multicolumn{2}{|l|}{ Pontuação Global } \\
\hline De 5-9 pontos & Situação social adequada ou aceitável \\
\hline De 10-14 pontos & Existe risco social \\
\hline Acima de 15 pontos & Existe problema social \\
\hline
\end{tabular}

Nota: Utilize o campo em branco para assinalar a alternativa correspondente ao contexto social relatado pela pessoa entrevistada. 


\section{DISCUSSÃO}

Importa enfatizar que a disponibilização adequada deste instrumento contribuirá para o planejamento de políticas públicas e ações junto à população idosa no Brasil em seus diferentes contextos sociais. O olhar ampliado sobre diferentes dimensões possibilita a organização e mobilização da rede social do idoso, prevenindo situações de fragilidade ou agravamento de seu quadro de saúde ${ }^{12,13}$.

Para a efetivação das traduções, os intérpretes discutiram aspectos técnicos e linguísticos de T1 e T2. O juiz neutro, em posse dessas traduções, construiu a síntese (T12) que referenciou as etapas seguintes. A composição de T12 atendeu aspectos de linguagem pautados no contexto avaliativo de cada componente da escala e na sequência foi retrotraduzida (RT1 e RT2). As versões RT1, RT2 e T12 foram avaliadas por um perito com conhecimento técnico da escala na versão espanhola. Sua avaliação apontou que T12 corresponde de forma consistente a proposta avaliativa da Escala de Avaliação Sociofamiliar em Idosos (Espanha).

Com base na proporção de concordância entre os especialistas frente aos critérios experimental

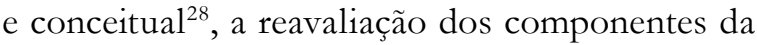
escala resultou em uma versão pré-teste adequada para avaliar a compreensão e manuseio por parte do entrevistador, bem como a compreensão do entrevistado. Com índice de concordância quase perfeito entre os especialistas, a avaliação das equivalências semântica, idiomática, experimental e conceitual, refletiu positivamente nos resultados decorrentes da aplicação da versão pré-teste.

As assertivas que compõem a escala na versão pré-teste (T12) foram lidas individualmente para cada um dos 30 participantes, que com unanimidade declararam boa compreensão.

A consistência interna global da versão préteste da escala, pela aferição dos seus domínios, apresentou um alfa de Cronbach de 0,77 , superior àqueles obtidos para as versões portuguesa $(0,41)^{14}$ e espanhola $(0,45)^{15}$, os quais denotam consistência interna razoável ${ }^{30}$.
Com finalidade mais discriminativa do que descritiva, os domínios avaliativos da EASFI medem aspectos distintos, o que pode reduzir a homogeneidade ${ }^{29}$. Para avaliação da condição sociofamiliar do indivíduo idoso, a escala considera por um lado, a situação familiar, relações sociais e suporte social e por outro lado, a situação econômica e as condições da habitação. Assim, a avaliação social abrange distintas dimensões compostas por diferentes variáveis de modo que não seria pertinente renunciar a qualquer dimensão avaliativa, mesmo que impacte no resultado da consistência interna.

Outro ponto que merece destaque está relacionado à aplicabilidade da escala. Alguns instrumentos têm sido usados para avaliar aspectos sociais em indivíduos de diferentes faixas etárias; contudo, se comparados à estrutura interna e operacional da EASFI, não englobam a função social em sua totalidade e apresentam alto grau de complexidade para o manuseio ${ }^{14}$. Nos estudos de validação realizados na Espanha ${ }^{15}$ e Portugal ${ }^{14}$, a escala foi preenchida pelo próprio participante. Para uso da EASFI no Brasil, sugere-se que a escala seja aplicada por um entrevistador para não reduzir a confiabilidade do instrumento.

O perfil de escolarização da população idosa no Brasil que reforça a sugestão de se adotar a aplicação da escala na modalidade face to face. Estudos demonstram que $50,2 \%$ da população residente no Brasil com idade superior a 60 anos frequentou a escola por até 4 anos e 30,7\% apresentaram menos de um ano de estudo ${ }^{33}$. A baixa escolarização foi também observada entre os entrevistados desta pesquisa. Assim sendo, o autopreenchimento, caso adotado no Brasil, poderia comprometer o resultado das avaliações.

A adaptação transcultural do instrumento para o contexto brasileiro é fundamental para a condução das demais etapas envolvidas na validação da EASFI. Essa escala em sua versão validada possibilitará o rastreio da situação sociofamiliar da pessoa idosa que vive no Brasil de um modo mais efetivo e contextualizado no que tange aos aspectos biopsicossociais do indivíduo ${ }^{34}$. 


\section{CONCLUSÃO}

Realizou-se a tradução e adaptação transcultural da Escala de Avaliação Sociofamiliar em Idosos (Espanha) para o contexto brasileiro. As avaliações das equivalências semântica, idiomática, cultural e conceitual, praticadas pelos membros do Comitê de Especialistas, tiveram um desfecho positivo, resultando na versão pré-teste da Escala de Avaliação Sociofamiliar em Idosos (Versão brasileira). A versão pré-teste apresentou boa consistência interna, com dados passíveis de reprodução. O estudo de validação deste instrumento está em andamento tendo por base essa versão pré-teste.

Editado por: Ana Carolina Lima Cavaletti

\section{REFERÊNCIAS}

1. Castro JLDC, Passos ALV, de Araújo LF, Santos JVDO. Análise psicossocial do envelhecimento entre idosos: as suas representações sociais. Actual Psicol. 2020;34(128):1-9.

2. Souza DS, Berlese DB, da Cunha GL, Cabral SM, dos Santos GA. Análise da relação do suporte social e da Síndrome de Fragilidade em idosos. Psicol Saúde Doenças. 2017;18(2):1-10.

3. Medina-Walpole A, Barker WH, Katz PR, Karuza J, Williams TF, Hall WJ. The current state of geriatric medicine: a national survey of fellowship-trained geriatricians, 1990 to 1998. J Am Geriatr Soc. 2002;50(5):1-10

4. de Sant'Ana LAJ, D’Elboux MJ. Suporte social e expectativa de cuidado de idosos: associação com variáveis socioeconômicas, saúde e funcionalidade. Saúde Debate. 2019;43(121):1-10.

5. World Health Organization. Ageing and life-course: Healthy Ageing and the Sustainable Development Goals. Geneva: WHO; 2020.

6. Barbosa GC, de Faria TK, Ribeiro PCC, Mármora $\mathrm{CHC}$. The relashionship between biopsychosocial and clinical outcomes of hospitalization, institutionalization and mortality according to the lifespan developement paradigma. Braz J Dev. 2020;6(11):1-10.

7. Carrapato P, Correia P, Garcia B. Determinante da saúde no Brasil: a procura da equidade na saúde. Saúde Soc. 2017;26(3):1-10.

8. Bueno EP. Envelhecer na sociedade contemporânea e o serviço social: perspectiva do trabalho profissional em rede de atenção à saúde da pessoa idosa. In: $16^{\circ}$ Congresso Brasileiro dos Assistentes Sociais - CBAS [Internet]. Brasília, DF; 30 out. - 03 set. de 2019. Brasília, DF: CBAS; 2019 [acesso em 01 maio 2021]. Disponível em: https://broseguini.bonino.com.br/ ojs/index.php/CBAS/article/view/335/333
9. de Carvalho IA, Epping-Jordan JA, Pot AM, Kelley E, Toro N, Thiyagarajan JA, et al. Organizing integrated health-care services to meet older people's needs. Bull WHO. 2017;95(11);1-10.

10. Partridge L, Deelen J, Slagboom PE. Facing up to the global challenges of ageing. Nature. 2018;561:1-10

11. Stich AK, Mozygemba K, Lysdahl KB, Pfadenhauer LM, Hofmann B, van der Wilt GJ, et al. Methods Assessing Sociocultural Aspects of Health Technologies: results of a Literature Review. Int J Technol Assess Health Care. 2019;35:1-10.

12. Maia RDS, Torres RA, Oliveira JGA, Maia EMC. Adaptação Transcultural de instrumentos para idosos no Brasil: uma revisão integrativa de literatura. Estud Interdiscipl Envelhec. 2014;19(2):1-10.

13. Rocha JA. O Envelhecimento humano e seus aspectos psicossociais. Rev Farol. 2018;6(6):1-10.

14. Mourão L. Aplicação da escala de Gijón em rastreio de risco social [Internet]. Portugal: Universidade de Aveiro; 2008 [acesso em 10 out. 2020]. Disponível em: https://ria.ua.pt/ bitstream/10773/3280/1/2009000424.pdf

15. Garcia Gonzalez J V, Diaz PE, Salamea GA, Cabrera GD, Menendez CA, Fernandez SA, et al. Evaluacion de la fiabilidad y validez de una escala de valoracion social en el anciano. Aten Prim. 1999;23(7):1-10.

16. Perú. Ministerio de Salud. Organización y Servicios de Salud. Resolución Ministerial. Norma Técnica de Salud para la Gestión de la Historia Clínica [Internet]. Perú: MS; 2018 [acesso em 25 out. 2020]. Disponível em: http://bvs.minsa.gob.pe/local/MINSA/4379.pdf

17. Barbosa KTF, Costa KNKM, Pontes MLF, Batista PSS, de Oliveira FMRL, Fernandes MGM. Envelhecimento e vulnerabilidade individual: um panorama dos idosos vinculados à estratégia saúde da família. Texto \& Contexto Enferm. 2017;26(2):1-10. 
18. de Jesus ITM, Diniz MAA, Lanzotti RB, Orlandi FS, Pavarin SCI, Zazzetta MS. Fragilidade e qualidade de vida de idosos em contexto de vulnerabilidade social. Texto \& Contexto Enferm. 2018;27(4):1-10.

19. de Melo LD, Arreguy-Sena C, Pinto PF, Gomes AMT, Santos JDC, Brandão MAG. Concepções de Idosos sobre as Políticas (Inter) Nacionais do Envelhecimento Humano. Rev Fam Ciclos Vida Saúde Contexto Soc. 2020;8(4):1-10.

20. Guedes MBOG, Lima KC, Caldas CP, Veras RP. Apoio social e o cuidado integral à saúde do idoso. Physis. 2017;27(4):1-10.

21. Domingues MA, Ordonez TN, Lima-Silva TB. Instrumentos de avaliação de rede de suporte social. In: Freitas EV, PY L, orgs. Tratado Brasileiro de Geriatria e Gerontologia. 4a ed. Rio de Janeiro: Guanabara-Koogan; 2016. p. 1570-7.

22. Domingues MA, Ordonez TN, Lima-Silva TB, Nagai PA, de Barros TC, Torres MJ. Revisão sistemática de instrumentos de avaliação de rede de suporte social para idosos. Rev Kairós Gerontol. 2013;15:1-10.

23. Torres LA, Rivera SM, Mendivelso FO, Yomayusa N, Cardenas HM, Hernandez C. Análisis del riesgo de deterioro funcional y sociofamiliar en el adulto mayor hospitalizado. Rev Méd Sanitas. 2019;22(1):1-10.

24. del Brutto OH, Mera RM, Recalde BY, Costa AF. Social Determinants of Health and Risk of SARSCoV-2 Infection in Community-Dwelling Older Adults Living in a Rural Latin American Setting. J Community Health. 2021;46(2):1-10.

25. Beltrán BE, Motta R, Gamarra MP, Araujo R, Castillo JJ. Comprehensive Geriatric Assessment in Elderly Patients with Aggressive Non-Hodgkin Lymphoma in Peru. Blood. 2018;132(Suppl 1):1-10.
26. Ardérius, M., Fournier, M., Alves, M., Bárrios, H., Duarte, M. J., Almeida, M. C. D. Nutritional Status as a Risk Factor in a Cohort of Geriatric Patients in a Specialized Convalescence Unit. Medicina Interna. 2020; 27(3), 4-9.

27. Beaton D, Bombardier C, Guillemin F, Ferraz MB. Recommendations for the cross-cultural adaptation of health status measures. New York Am Acad Orthop Surg. 2002;12:1-9.

28. Alexandre NMC, Coluci MZO. Validade de conteúdo nos processos de construção e adaptação de instrumentos de medidas. Ciênc Saúde Colet. 2011;16(17):3061-8.

29. de Souza AC, Alexandre NMC, Guirardello EB. Propriedades psicométricas na avaliação de instrumentos: avaliação da confiabilidade e da validade. Epidemiol Serv Saúde. 2017;26(3):1-10.

30. Amirrudin M, Nasution K, Supahar S. Effect of Variability on Cronbach Alpha Reliability in Research Practice. J Mat Stat Komputasi. 2020;17(2):223-30.

31. Coluci MZO, Alexandre NMC, Rosecrance J. Reliability and validity of an ergonomics-related Job Factors Questionnaire. Int J Ind Ergon. 2009;39(6):1-10.

32. Landis JR, Koch GG. The Measurement of Observer Agreement for Categorical Data. Biometrics. 1977;33(1):1-10.

33. Travassos GF, Coelho AB, Arends-Kuenning MP. The elderly in Brazil: Demographic transition, profile, and socioeconomic condition. Rev Bras Estud Popul. 2020;37:1-10.

34. Fletcher AE, Jones DA, Bulpitt CJ, Tulloch AJ. The MRC trial of assessment and management of older people in the community: objectives, design and interventions [ISRCTN23494848]. BMC Health Serv Res. 2002;2(1):1-10. 